\title{
IMMUNISATION ADVERSE EVENTS CLINICS
}

\section{Nicholas Wood}

National Centre for Immunisation Research and Surveillance of Vaccine Preventable Diseases The University of Sydney and The Children's Hospital at Westmead

As the incidence of vaccine preventable diseases has declined, and more new vaccines are marketed, adverse events following immunisation have attracted greater attention from the public and from health care providers. At a population level, post-licensure monitoring of vaccine safety in many countries relies on notifications to passive surveillance systems, such as the Australian Adverse Drug Reactions Advisory Committee (ADRAC). Australia is better supplied with services to investigate and manage adverse events in individual patients through special immunisation services than most comparable countries. This article provides a brief background to the establishment of clinics that address adverse events following immunisation (AEFI), and describes the work of the Immunisation Adverse Events Clinic at The Children's Hospital at Westmead.

Vaccine-related adverse events clinics were first established in England in the 1980s, ${ }^{1-4}$ following a major fall in the uptake of whole-cell pertussis vaccines (DTPw) after adverse publicity. ${ }^{2}$ Similar clinics were set up in several Australian centres from 1994 onwards. ${ }^{5}$ At first these clinics mainly addressed concerns about completing
DTPw schedules in the context of a resurgence of pertussis..$^{6,8}$ In 1996, the Immunisation Adverse Events Clinic started at The Children's Hospital at Westmead. The first group of such clinics in the USA, called Clinical Immunization Safety Assessment Centers (CISA) was established in October 2001. ${ }^{9}$

\section{IMMUNISATION ADVERSE EVENTS CLINIC, WESTMEAD}

\section{Referral}

The Immunisation Adverse Events Clinic, Westmead, accepts referrals of children from health professionals, after screening by a clinical nurse consultant. The clinic is held fortnightly and is staffed by a consultant paediatrician, a paediatrician-in-training and a clinical nurse consultant.

Children who may be referred to the clinic are likely to have experienced one of the following adverse events after a previous vaccination: anaphylactoid reaction, encephalopathy, convulsion, very severe local reaction, severe hypotonic hyporesponsive episode (HHE), or a condition requiring hospitalisation after vaccination. Children with pre-existing medical conditions where vaccination could be contraindicated are also seen. However, parents who are reluctant to vaccinate a child, or who simply have questions about vaccination, should see a general practitioner in the first instance.

\section{FIGURE 1}

NUMBER OF ATTENDANCES AT THE WESTMEAD IMMUNISATION ADVERSE EVENTS CLINIC, 1999-2001

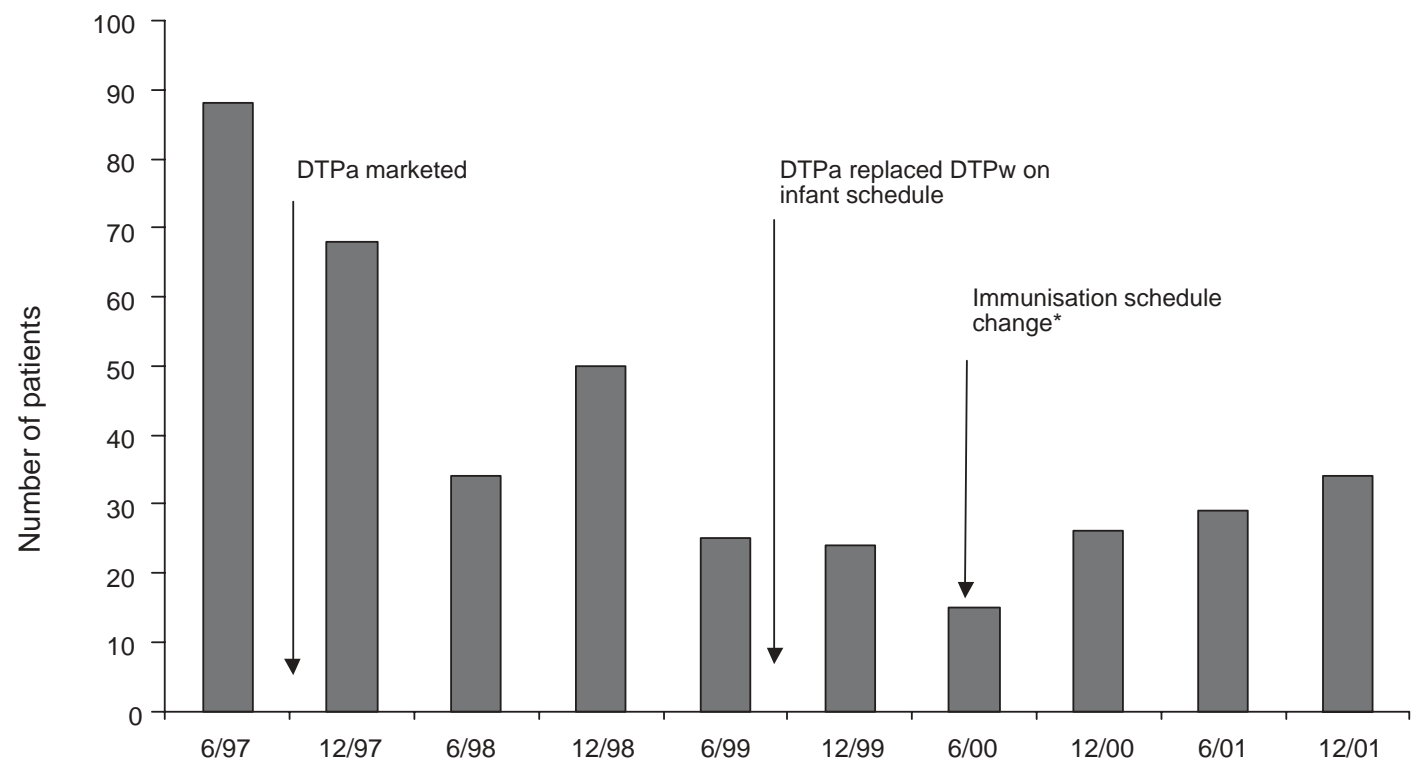

Date attending clinic by half year

* indicates schedule change: introduction of universal hepatitis B vaccine (including a birth dose) and use of DTPa-hepatitis B combination vaccine. 


\section{Case 1}

A nine-month old male infant was referred to the Clinic by his paediatrician for advice about his vaccinations, which were due at six months of age. Within 12 hours of the four-month vaccination with DTPa-HepB, Hib and oral polio vaccine, the infant had developed fever, lethargy, poor feeding, abnormal behaviour, and a papular erythematous rash. His parents brought him to the emergency department of The Children's Hospital at Westmead. Investigations revealed a monocytic cerebrospinal fluid (CSF) pleocytosis, slightly elevated CSF protein, reduced CSF glucose, and peripheral blood pleocytosis consistent with a diagnosis of viral meningoencephalitis. Two weeks after this episode he had completely recovered. Although no virus was isolated, the clinical course and CSF results are consistent with a coincidental viral meningoencephalitis, unrelated to his four-month vaccinations. After discussion with his parents, the infant was admitted overnight and given his routine six-month vaccinations without any AEFI.

\section{Case 2}

A five-month old female infant, who had been born prematurely, was referred to the Clinic by her general practitioner. She was born by emergency caesarean section at 28 weeks' gestation because of maternal pregnancy-induced hypertension. In the 24-hour period following her two-month (36 weeks corrected gestational age) vaccinations she experienced 1215 apnoeas and bradycardias. She was nursed in increased oxygen for the first 24 hours and then in room air without subsequent problems; investigations revealed no cause for the apnoea.

She was admitted to The Children's Hospital at Westmead as a day-stay patient, aged five months, for her routine four-month vaccinations, which were tolerated without any AEFI.

\section{Case 3}

An eight-month old female was referred to the Clinic by her general practitioner. Following her two-month vaccinations, she had been irritable and hypersensitive to external stimuli, with high-pitched screaming for one hour. The symptoms persisted for 48 hours, after which she recovered completely. At 4.5 months of age, multiple protein intolerance and chemical insensitivity, managed with a hypoallergenic diet, were diagnosed.

When she was eight months old, she was admitted to The Children's Hospital at Westmead, as a day-stay patient for her routine four-month vaccinations, which were tolerated without recurrence of the adverse event; a catch-up schedule was recommended.
The parents of children seen at the Clinic are counselled about continuation of the standard vaccination schedule. Children may be vaccinated under supervision at the Clinic or admitted later as day-stay or overnight cases to be vaccinated with a longer period of observation. Such inpatient observation has proved very useful in encouraging re-vaccination after previous convulsions, HHEs, or apparent life-threatening events. ${ }^{10}$ Children vaccinated at the Immunisation Adverse Events Clinic are routinely followed-up with a telephone interview 72 hours later, to ascertain any AEFI that may have occurred after vaccination.

\section{Attendances}

Between January 1997 and October 2002, the Clinic reviewed 453 patients, 40 per cent of whom had an underlying medical illness, such as asthma or atopy, or a developmental delay. Most of these patients (364/453 or 80 per cent) had had a previous suspected AEFI; the remaining 20 per cent were predominantly seen for advice about future vaccination: for example, MMR vaccination in the presence of an allergy to eggs.

The most common adverse events were fever, screaming, or severe local reactions, and most were associated with combinations of vaccines that included diphtheria, tetanus, and pertussis antigens-particularly combinations containing DTPw. Children who had experienced more severe adverse events, such as convulsions, HHEs, and encephalopathies, were also seen; in most cases they were able to continue their immunisation schedule. ${ }^{11}$ The change from whole-cell to acellular pertussis vaccine (DTPa) in 1999 resulted in a fall in the number of referrals to the clinic of children with adverse events commonly associated with DTPw—such as fever, inconsolable crying, HHEs, and seizures (Figure 1). ${ }^{12}$ Examples of cases are presented in Table 1.

\section{DISCUSSION}

The importance of adverse events following immunisation should not be underestimated. In the 1995 Australian national survey on childhood immunisation, carried out by the Australian Bureau of Statistics, 6.6 per cent of parents cited concerns about side-effects as reasons for not vaccinating their children. Changes to the immunisation schedule, introduction of new vaccines, and a tendency for the media to focus on adverse events increase both provider and parental concern. ${ }^{13}$ While a telephone hotline can provide detailed information and a 
response to queries and anxiety, ${ }^{14}$ a personal consultation enables physical examination of the patient, detailed discussion of the pros and cons of continuing the vaccination series, discussion of written educational material, and re-vaccination if indicated. Information about vaccination, often anecdotal and misleading, is freely available on the Internet, ${ }^{15}$ and the special clinics provide a means for parents to validate concerns and resolve any misconceptions about adverse events.

At a population level, special immunisation adverse events clinics can enhance surveillance of adverse events after immunisation in a number of ways that are described below.

\section{Provision of clinical data}

Adverse events seen at the Westmead Clinic are reported to the NSW Department of Health using the standardised 'blue' reporting form. Accumulated data from the Australian Adverse Drug Reactions Advisory Committee (ADRAC) can identify reactions that occur infrequently and which may be associated with vaccination, and can monitor for unusually high rates of adverse or previously undocumented events.

\section{Development of case definitions and clinical protocols} The collection of detailed clinical data on AEFIs and collaboration with other centres enable case definitions of adverse events following immunisation to be developed. ${ }^{16}$ Similarly, clinical protocols can be formulated to standardise the management of adverse events. The clinics are also a site for long-term neurodevelopmental follow-up of children who have experienced an AEFI.

\section{Detailed investigation of adverse events}

Evaluation of patients with similar adverse events can help determine genetic or other risk factors for these adverse events, including identification of possible serological and cellular markers predictive of adverse events.

\section{Demonstration of the safety of re-vaccination after severe adverse events}

Several reports have illustrated the effectiveness of these clinics in promoting the continuation of the immunisation schedule when vaccines would otherwise have been missed. ${ }^{1,3-5,10}$ In particular, children with a history of severe AEFIs have been successfully re-vaccinated., ${ }^{5,10,11}$ The clinics also have a role in dissemination of this information to a wider health provider audience.

\section{THE FUTURE}

The role of immunisation adverse event clinics will evolve, as the incidence of diseases prevented by vaccination declines further; as new vaccines and methods of administration are licensed; and as adverse events following immunisation assume greater significance. The clinics demonstrate to providers and parents that adverse events are taken seriously but rarely contraindicate further doses of vaccine.

For more information contact the Immunisation Clinical Nurse Consultant, Immunisation Adverse Events Clinic, The Children's Hospital at Westmead, by telephone on (02) 98452113.

\section{REFERENCES}

1. Newport MJ, Conway SP. Experience of a specialist service for advice on childhood immunisation. J Infect 1993;26:295-300.

2. Lingam S, Miller CL, Pateman J. Role of an immunisation advisory clinic. Br Med J 1986;292:939-40.

3. Ko ML, Rao M, Teare L, et al. Outcome of referrals to a district immunisation advisory clinic. Commun Dis Rep CDR Rev 1995;5:R146-9.

4. Hall R, Williams A. Special advisory service for immunisation. Arch Dis Child 1988;63: 1498-1500.

5. Andrews RM, Kempe AE, Sinn KK, Herceg A. Vaccinating children with a history of serious reactions after vaccination or of egg allergy. Med J Aust 1998;168:491-4.

6. Burgess MA, McIntyre PB, Heath TC. Pertussis re-emerging: who is responsible? Aust N Z J Public Health 1998;22:9-10.

7. Gangarosa EJ, Galazka AM, Wolfe CR, et al. Impact of the anti-vaccine movements on pertussis control: the untold story. Lancet 1998;351:356-61.

8. Andrews R, Herceg A, Roberts C. Pertussis notifications in Australia, 1991 to 1997. Commun Dis Intell 1997;21:145-8.

9. Pless R, Casey C, Chen R. Clinical Immunization Safety Assessment Centers: Improving the Evaluation, Management and Understanding of Adverse Events Possibly Related to Immunizations. National Immunization Program, Centers for Disease Control (CDC). www.partnersforimmunization.org/ cisa.pdf. Accessed 2 August 2002.

10. Goodwin H, Nash M, Gold M, et al. Vaccination of children following a previous hypotonic-hyporesponsive episode. $J$ Paediatr Child Health 1999;35:549-52.

11. Gold M, Goodwin H, Botham S, et al. Re-vaccination of 421 children with a past history of an adverse reaction in a special immunisation service. Arch Dis Child 2000;83:128-31.

12. Wood N, Wang H, Codarini M, et al. The changing role of a special immunisation clinic - the Sydney experience post 1999 [poster presentation]. Public Health Australia Conference 2002, Melbourne, Victoria, Australia.

13. Leask J, Chapman S. An attempt to swindle nature: press anti-immunisation reportage 1993-1997. Aust N Z J Public Health 1998;22:17-27.

14. Finlay F, McKechnie L, Pearce A, Lenton S. Immunization telephone hotline audit. Ambulatory Child Health 1999;5:295-302.

15. Davies P, Chapman S, Leask J. Antivaccination activists on the World Wide Web. Arch Dis Child 2002;87:22-5.

16. Bonhoeffer J, Kohl K, Chen R. The Brighton Collaboration: addressing the need for standardized case definitions of adverse events following immunization (AEFI). Vaccine 2002;21:298-302. $\mathbb{W}$ 